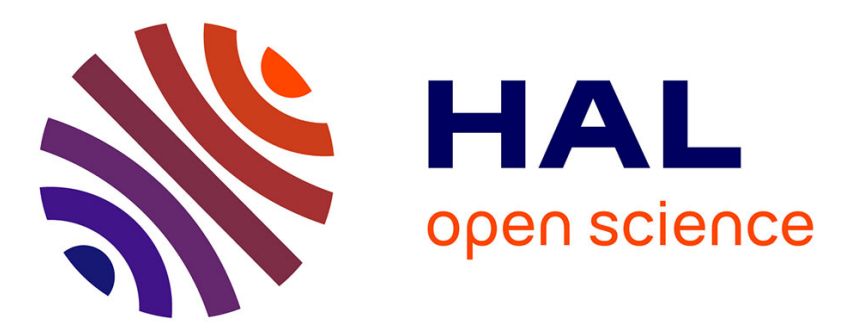

\title{
Stabilization of coupled linear heterodirectional hyperbolic PDE-ODE systems
}

Florent Di Meglio, Federico Bribiesca Argomedo, Long Hu, Miroslav Krstic

\section{To cite this version:}

Florent Di Meglio, Federico Bribiesca Argomedo, Long Hu, Miroslav Krstic. Stabilization of coupled linear heterodirectional hyperbolic PDE-ODE systems. Automatica, 2018, 87, pp.281-289. 10.1016/j.automatica.2017.09.027 . hal-01376564v2

HAL Id: hal-01376564

https://hal.science/hal-01376564v2

Submitted on 14 Feb 2017

HAL is a multi-disciplinary open access archive for the deposit and dissemination of scientific research documents, whether they are published or not. The documents may come from teaching and research institutions in France or abroad, or from public or private research centers.
L'archive ouverte pluridisciplinaire HAL, est destinée au dépôt et à la diffusion de documents scientifiques de niveau recherche, publiés ou non, émanant des établissements d'enseignement et de recherche français ou étrangers, des laboratoires publics ou privés. 


\title{
Stabilization of coupled linear heterodirectional hyperbolic PDE-ODE systems
}

\author{
Florent Di Meglio ${ }^{\mathrm{a}}$, Federico Bribiesca Argomedo ${ }^{\mathrm{b}}$, Long Hu$^{\mathrm{c}}$, Miroslav Krstic ${ }^{\mathrm{d}}$ \\ ${ }^{\mathrm{a}}$ CAS - Centre automatique et systèmes, MINES ParisTech, PSL Research University, 60 bd St Michel, 75006 Paris, France \\ ${ }^{\mathrm{b}}$ Université de Lyon - Laboratoire Ampère (CNRS UMR5005) - INSA de Lyon, 69621 Villeurbanne CEDEX, France \\ ${ }^{\mathrm{c}}$ Fudan University, 220 Handan Rd, Yangpu, Shanghai, China \\ ${ }^{\mathrm{d}}$ Department of Mechanical $\mathcal{F}$ Aero. Eng., University of California, San Diego, La Jolla, CA 92093-0411
}

\begin{abstract}
We solve the problem of stabilizing a linear ODE having a system of a linearly coupled hyperbolic PDEs in the actuating and sensing paths. The system is exponentially stabilized by mapping it to a target system with a cascade structure using a Volterra transformation.
\end{abstract}

Key words: Stabilization, Distributed Parameter Systems

\section{Introduction}

The interest for coupled Ordinary Differential EquationsPartial Differential Equations (ODE-PDE) systems has first emerged when considering delays in the actuating and sensing paths of ODE. Delays can be seen as first-order hyperbolic PDEs. There are many approaches to deal with input or measurement delays, usually divided into two categories: memoryless controllers, which extend standard control techniques without explicitly accounting for the delay in the control design $[16,25,9]$; and prediction-based controllers aiming at explicitly compensating the delay [20,4,2].

The use of Lyapunov and backstepping methods enabled dealing with more involved PDEs in the actuating and sensing paths. In [13], an output feedback control law is derived for an ODE having a heat equation in the actuating and sensing paths. The coupled PDE-ODE system is stabilized using an observer-controller structure relying on a backstepping approach. The same approach has been used to deal with ODEs coupled (rather than cascaded) with parabolic PDEs [21], uncertain parabolic PDEs [15], or ODE-Schrödinger cascades [17]. Lyapunov methods enable the design of static output feedback controllers for nonlinear ODE-parabolic PDE cascades, as in [24].

The first application of the backstepping approach to deal with hyperbolic PDE-ODE couplings is [14] where actuator and sensor delays are explicitly compensated. While this problem had already been tackled by, e.g., the Smith predictor [20], the reformulation of the delay as a linear ODE enabled numerous related problem to be tackled, most notably non-constant and uncertain delays [2,4]. In [22], the problem of stabilizing a multi-input ODE with distinct delays is tackled using a backstepping approach. In [6], an observer is designed for an ODE having a homodirectional ${ }^{1}$ hyperbolic PDE in the sensing path, relying on a Lyapunov analysis requiring to solve Linear Matrix Inequalities (LMI). As will appear, the systems of [22] are particular cases of the system considered here, although the control design approaches are different.

Here, we solve the problem of stabilizing an ODE with a system of first-order linear hyperbolic PDEs in the sensing

\section{Email addresses:}

florent.di_meglio@mines-paristech.fr (Florent Di Meglio), federico.bribiesca@insa-lyon.fr (Federico Bribiesca Argomedo), longhu@ipm6.fr (Long Hu), krstic@ucsd.edu (Miroslav Krstic).

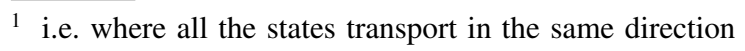




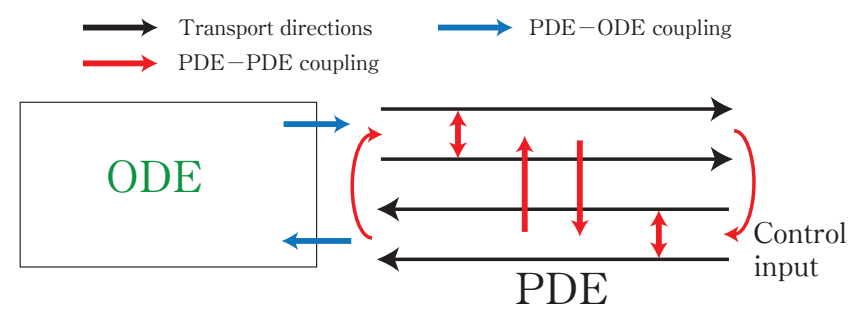

Fig. 1. Schematic view of the ODE-PDE coupling structure

and actuating paths, i.e. we consider the following system

$$
\begin{aligned}
\dot{X}(t) & =A X(t)+B v(t, 0) \\
u_{t}(t, x) & =-\Lambda^{+} u_{x}(t, x)+\Sigma^{++} u(t, x)+\Sigma^{+-} v(t, x) \\
v_{t}(t, x) & =\Lambda^{-} v_{x}(t, x)+\Sigma^{-+} u(t, x)+\Sigma^{--} v(t, x) \\
u(t, 0) & =Q_{0} v(t, 0)+C X(t) \\
v(t, 1) & =R_{1} u(t, 1)+U(t)
\end{aligned}
$$

where $t>0$ and $x \in[0,1]$ are respectively the time and space variables, $X \in \mathbb{R}^{p}$ is the ODE state, $u(t, x) \in \mathbb{R}^{n}$ and $v(t, x) \in$ $\mathbb{R}^{m}$ are the PDE states and $U(t)$ is the control input. The matrices $\Lambda^{+}$and $\Lambda^{-}$are such that

$$
\Lambda^{+}=\operatorname{diag}\left(\lambda_{1}, \ldots, \lambda_{n}\right), \quad \Lambda^{-}=\operatorname{diag}\left(\mu_{1}, \ldots, \mu_{m}\right)
$$

with

$$
-\mu_{1}<\cdots<-\mu_{m}<0<\lambda_{1}<\cdots<\lambda_{n}
$$

The system naturally features several feedback loops or couplings that can be sources of instabilities:

- Inside the ODE itself (the $A$ matrix in Equation (1))

- Coupling between hyperbolic states inside the spatial domain (the $\Sigma{ }^{*}$ matrices in (2),(3))

- Coupling between hyperbolic states at the boundary (the $Q_{0}$ and $R_{1}$ matrices in (4),(5))

- Coupling between the PDE and the ODE (the $B$ and $C$ matrices in (1),(4))

- A combination of all the above.

This structure is schematically depicted on Figure 1. This problem is motivated by applications in the drilling industry, more precisely the suppression of mechanical vibrations. Drilling systems are composed of long flexible strings subject to axial and torsional vibrations that propagate upwards and downwards. At the bottom end, the so-called drill bit crushes rock to create the borehole and is subject to friction and cutting forces. The ODE state $X$ then corresponds to the drill bit axial and torsional positions while the PDE states represent the propagation of torsional and axial waves from and to the drill bit.

When damping of the vibrations along the drillstring is neglected and the axial and torsional vibrations are coupled, the PDE reduces to two delay equations. Several contributions have taken advantage of this simplification and designed stabilizing feedback laws, e.g. relying on neutral system approaches [19], flatness approaches [18] or predictor-based approaches $[3,5]$. However, no existing solution simultaneously allows stabilization

- taking into account damping inside the PDE domain

- for a model of both axial and torsional vibrations, yielding 4 coupled PDE states rather than two delay equations $[7,10]$.

Here, we solve these problems within the general setting of Equations (1)-(5). The system is mapped to an exponentially stable target system using a Volterra transformation. The target system has a cascade structure ensuring its convergence to the zero equilibrium. The design is based on a recent result on heterodirectional systems of hyperbolic PDEs [12].

The paper is organized as follows. In Section 2 we present the backstepping control design. In Section 3 we present a general well-posedness result for a class of hyperbolic PDEs on a triangular domain. In Section 4 we apply these results to the considered problem and state the main result. We conclude in Section 5 with perspectives for future work.

\section{Control design}

The control design is based on a Volterra transformation mapping the state $(X, u, v)$ to a target system $(X, \alpha, \beta)$ with desirable properties. The target system equations are described in the next section.

\subsection{Target system}

We design the target system as follows

$$
\begin{aligned}
\dot{X}(t)= & (A+B \mathcal{K}) X(t)+B \beta(t, 0) \\
\alpha_{t}(t, x)= & -\Lambda^{+} \alpha_{x}(t, x)+\Sigma^{++} \alpha(t, x)+\Sigma^{+-} \beta(t, x)+D(x) X(t) \\
& +\int_{0}^{x} C^{+}(x, y) \alpha(t, y) d y+\int_{0}^{x} C^{-}(x, y) \beta(t, y) d y \\
\beta_{t}(t, x)= & \Lambda^{-} \beta_{x}(t, x)+G(x) \beta(t, 0)+\bar{\Sigma} \beta(t, x) \\
\alpha(t, 0)= & Q_{0} \beta(t, 0)+C_{0} X(t) \\
\beta(t, 1)= & 0
\end{aligned}
$$

where $C_{0}, C^{+}, C^{-}$and $D$ have yet to be defined and $G(\cdot)$ and $\bar{\Sigma}$ are defined as

$$
G(x)=\left(\begin{array}{cccc}
0 & \cdots & \cdots & 0 \\
g_{2,1}(x) & \ddots & \ddots & \vdots \\
\vdots & \ddots & \ddots & \vdots \\
g_{m, 1}(x) & \cdots & g_{m, m-1} & 0
\end{array}\right), \quad \bar{\Sigma}=\operatorname{diag}\left(\Sigma^{--}\right)
$$




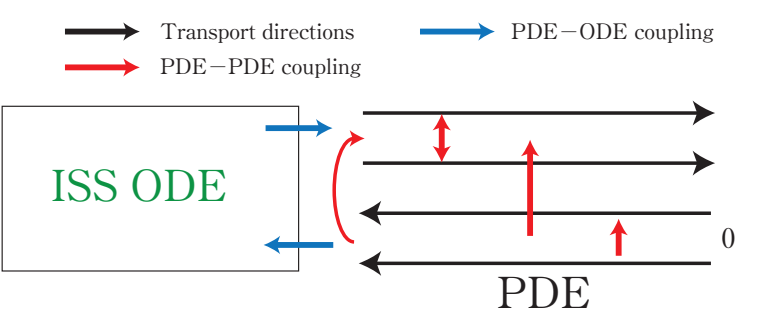

Fig. 2. Schematic view of the cascade structure of the target system.

The target system has a cascade structure schematically depicted on Figure 2. Its stability properties are assessed in the following Lemma.

Lemma 1 Denote $\mathcal{T}=\{0 \leq y \leq x \leq 1\}$. Under the following assumptions

(i) $A+B \mathcal{K}$ is Hurwitz

(ii) $C^{+}, C^{-} \in L^{\infty}(\mathcal{T})$

(iii) $G, D \in L^{\infty}([0,1])$

the zero equilibrium of System (8)-(12) is exponentially stable in the $L^{2}$ sense.

Proof Consider the following Lyapunov functional

$$
\begin{aligned}
& V(t)=\int_{0}^{1} e^{-\delta x} \alpha(t, x)^{\top}\left(\Lambda^{+}\right)^{-1} \alpha(t, x) d x \\
& +\int_{0}^{1} e^{\delta x} \beta(t, x)^{\top}\left(\Lambda^{-}\right)^{-1} R \beta(t, x) d x+X(t)^{\top} P X(t)
\end{aligned}
$$

where the symmetric definite positive matrix $P$, the diagonal matrix $R=\operatorname{diag}\left(r_{1}, \ldots, r_{m}\right)$ and the design parameter $\delta>0$ are yet to be determined. Differentiating with respect to time and integrating by parts yields

$$
\begin{gathered}
\dot{V}(t)=\left[-e^{-\delta x} \alpha(t, x)^{\top} \alpha(t, x)+e^{\delta x} \beta(t, x)^{\top} R \beta(t, x)\right]_{0}^{1} \\
-\int_{0}^{1} \delta e^{-\delta x} \alpha(t, x)^{\top} \alpha(t, x) d x-\int_{0}^{1} \delta e^{\delta x} \beta(t, x)^{\top} R \beta(, x) d x \\
+2 \int_{0}^{1} e^{-\delta x} \alpha(t, x)^{\top}\left(\Lambda^{+}\right)^{-1}\left[\Sigma^{++} \alpha(t, x)\right. \\
+\Sigma^{+-} \beta(t, x)+D(x) X(t) \\
\left.+\int_{0}^{x} C^{+}(x, y) \alpha(t, y) d y+\int_{0}^{x} C^{-}(x, y) \beta(t, y) d y\right] d x \\
+2 \int_{0}^{1} e^{\delta x} \beta(t, x)^{\top}\left(\Lambda^{-}\right)^{-1} R[G(x) \beta(t, 0)+\bar{\Sigma} \beta(t, x)] d x \\
+X(t)^{\top}\left[P(A+B \mathcal{K})+(A+B \mathcal{K})^{\top} P\right] X(t) \\
+X(t)^{\top}\left(P B+B^{\top} P\right) \beta(t, 0)
\end{gathered}
$$

Using Young's inequality yields the existence of $M, \gamma>0$ such that

$$
\begin{aligned}
2 \int_{0}^{1} e^{-\delta x} \alpha(t, x)^{\top}\left(\Lambda^{+}\right)^{-1} \Sigma^{++} \alpha(t, x) d x \\
\leq M \int_{0}^{1} e^{-\delta x} \alpha(t, x)^{\top}\left(\Lambda^{+}\right)^{-1} \alpha(t, x) d x
\end{aligned}
$$

$$
\begin{gathered}
2 \int_{0}^{1} e^{-\delta x} \alpha(t, x)^{\top}\left(\Lambda^{+}\right)^{-1} \Sigma^{+-} \beta(t, x) d x \\
\leq M\left(\int_{0}^{1} e^{-\delta x} \alpha(t, x)^{\top} R^{-1} \alpha(t, x) d x\right. \\
\left.\quad+\int_{0}^{1} e^{\delta x} \beta(t, x)^{\top} R \beta(t, x) d x\right)
\end{gathered}
$$

$$
\begin{array}{r}
2 \int_{0}^{1} \int_{0}^{x} e^{-\delta x} \alpha(t, x)^{\top}\left(\Lambda^{+}\right)^{-1} C^{+}(x, y) \alpha(t, y) d x d y \\
\leq \frac{M}{\delta} \int_{0}^{1} e^{-\delta x} \alpha(t, x)^{\top}\left(\Lambda^{+}\right)^{-1} \alpha(t, x) d x
\end{array}
$$

$$
\begin{array}{r}
2 \int_{0}^{1} \int_{0}^{x} e^{-\delta x} \alpha(t, x)^{\top}\left(\Lambda^{+}\right)^{-1} C^{-}(x, y) \beta(t, y) d x d y \\
\leq \frac{M}{\delta}\left(\int_{0}^{1} e^{-\delta x} \alpha(t, x)^{\top} R^{-1} \alpha(t, x) d x\right. \\
\left.+\int_{0}^{1} e^{\delta x} \beta(t, x)^{\top} R \beta(t, x) d x\right)
\end{array}
$$

$$
\begin{aligned}
& \int_{0}^{1} e^{-\delta x} \alpha(t, x)^{\top}\left(\Lambda^{+}\right)^{-1} D(x) X(t) d x \\
& \leq M\left[\frac{1}{\gamma}|X(t)|+\gamma \int_{0}^{1} e^{-\delta x} \alpha(t, x)^{\top}\left(\Lambda^{+}\right)^{-1} \alpha(t, x) d x\right] \\
& X(t)^{\top}\left(P B+B^{\top} P\right) \beta(t, 0) \leq M\left[\frac{1}{\gamma}|X(t)|+\gamma \beta(t, 0)^{\top} \beta(t, 0)\right]
\end{aligned}
$$

where $\gamma>0$ is a design parameter to be defined. Further, given the structure of the $G$ matrix given by (13), there exists $M>0$ such that

$$
\begin{aligned}
& \int_{0}^{1} e^{\delta x} \beta(t, x)^{\top}\left(\Lambda^{-}\right)^{-1} R G(x) \beta(t, 0) d x \\
& \leq M\left[\int_{0}^{1} e^{\delta x} \beta(t, x)^{\top} R \beta(t, x) d x+e^{\delta} \beta(t, 0)^{\top} C \beta(t, 0)\right]
\end{aligned}
$$


where

$$
C=\operatorname{diag}\left(c_{1}, \cdots, c_{m}\right), \quad c_{i}= \begin{cases}\sum_{j=i+1}^{m} r_{j}, & 1 \leq i \leq m-1 \\ 0, & i=m\end{cases}
$$

Besides, let $S=\operatorname{diag}\left(s_{1}, \ldots, s_{m}\right)$ such that

$$
Q_{0}^{\top} Q_{0}-S<0
$$

Finally, plugging (8),(11),(12) into (15) and denoting $Q=$ $-\left[P(A+B \mathcal{K})+(A+B \mathcal{K})^{\top} P\right]>0$ yields

$$
\begin{gathered}
\dot{V}(t) \leq-\beta(t, 0)^{\top}\left[R-M C e^{\delta}-\gamma M I_{m \times m}-S\right] \beta(t, 0) \\
-X(t)^{\top}\left[Q-\frac{2}{\gamma} I_{p \times p}\right] X(t) \\
-\int_{0}^{x} e^{-\delta x} \alpha(t, x)^{\top}\left[(\delta-\gamma) I_{n \times n}-M\left(1+\frac{1}{\delta}\right)\left(I_{n \times n}+R^{-1}\right)\right] \alpha(t, x) d x \\
-\left[\delta-M\left(2+\frac{1}{\delta}\right)\right] \int_{0}^{1} e^{\delta x} \beta(t, x)^{\top} R \beta(t, x) d x
\end{gathered}
$$

thus, picking $\gamma, R$ such that

$$
\begin{gathered}
Q-\frac{2}{\gamma} I_{p \times p} \prec 0 \\
\forall i=1, \ldots, m-1 \quad r_{i}>M e^{\delta} \sum_{j=i+1}^{m} r_{j}+\gamma M+s_{i} \\
r_{m}>\gamma M+s_{m}
\end{gathered}
$$

and $\delta$ large enough concludes the proof.

\subsection{Volterra Transform}

To map the original system (1)-(5) to the target system (8)(12), we use the following Volterra transformation

$$
\begin{aligned}
\alpha(t, x) \equiv u(t, x) & \\
\beta(t, x)= & v(t, x)-\int_{0}^{x} K(x, y) u(t, y) d y \\
& -\int_{0}^{x} L(x, y) v(t, y) d y-\gamma(x) X(t)
\end{aligned}
$$

where the kernels $K, L$ and $\gamma$ have yet to be defined. Differentiating (30) w.r.t. space and time yields the following kernel equations

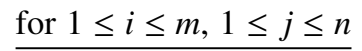

$$
\begin{aligned}
\mu_{i} \partial_{x} K_{i j}(x, y)- & \lambda_{j} \partial_{y} K_{i j}(x, y)=-\sigma_{i i}^{--} K_{i j}(x, y) \\
& +\sum_{k=1}^{n} \sigma_{k j}^{++} K_{i k}(x, y)+\sum_{k=1}^{m} \sigma_{k j}^{-+} L_{i k}(x, y)
\end{aligned}
$$

for $1 \leq i \leq m, 1 \leq j \leq m$

$$
\begin{aligned}
\mu_{i} \partial_{x} L_{i j}(x, y)+ & \mu_{j} \partial_{y} L_{i j}(x, y)=-\sigma_{i i}^{--} L_{i j}(x, y) \\
& +\sum_{k=1}^{m} \sigma_{k j}^{--} L_{i k}(x, y)+\sum_{k=1}^{n} \sigma_{k j}^{+-} K_{i k}(x, y)
\end{aligned}
$$

along with the following set of boundary conditions, for $i=$ $1, \ldots, m$

$$
\begin{aligned}
\forall j, \quad K_{i j}(x, x) & =-\frac{\sigma_{i j}^{-+}}{\mu_{i}+\lambda_{j}} \\
\forall j \neq i, \quad L_{i j}(x, x) & =-\frac{\sigma_{i j}^{--}}{\mu_{i}-\mu_{j}} \triangleq l_{i j} \\
\forall j \geq i \quad \mu_{j} L_{i j}(x, 0) & =\sum_{k=1}^{n} \lambda_{k} K_{i k}(x, 0) q_{k, j}+\sum_{k=1}^{p} b_{k j} \gamma_{i k}(x)
\end{aligned}
$$

where the $q_{k, j}$ in (35) are the elements of $Q_{0}$. Besides, $\gamma$ satisfies the following ODE

$$
\begin{aligned}
\forall j, \quad \mu_{i} \gamma_{i j}^{\prime}(x) & =\sum_{k=1}^{p}\left[a_{k j}+\sum_{\substack{l=1 \\
l<i}}^{m} b_{k l} \kappa_{l j}\right] \gamma_{i k}(x)-\sigma_{i i}^{--} \gamma_{i j}(x) \\
& -\sum_{\substack{k=1 \\
k<i}}^{m} \mu_{k} L^{i k}(x, 0) \kappa_{k j} \\
& +\sum_{k=1}^{n} \lambda_{k}\left[c_{k j}+\sum_{\substack{l=1 \\
l<i}}^{m} q_{k l} \kappa_{l j}\right] K^{i k}(x, 0)
\end{aligned}
$$

with initial condition

$$
\forall j, \quad \gamma_{i j}(0)=\kappa_{i j}
$$

where the $\kappa_{i j}$ are the entries of the control matrix gain $\mathcal{K}$. To ensure well-posedness of the system, we add the following arbitrary boundary conditions

$$
\forall j<i, \quad L_{i j}(1, x)=l_{i j}(x)
$$

These are degrees of freedom in the control design. However, their effect on the closed-loop performances are still unclear, thus, to study well-posedness, which we do in the next section, we only impose $l_{i j} \in L^{\infty}([0,1])$. Besides, provided the $K$ and $L$ kernels are well-posed, the coefficients of $G, C_{0} C^{+}, C^{-}$and $D$ are given by

$$
\begin{array}{r}
\forall j<i, \quad g_{i j}(x)=\mu_{j} L_{i j}(x, 0)-\sum_{k=1}^{n} \lambda_{k} K_{i k}(x, 0) q_{k, j} \\
-\sum_{k=1}^{p} b_{k j} \gamma_{i k}(x)
\end{array}
$$




$$
\begin{aligned}
C^{+}(x, y) & =\Sigma^{+-} K(x, y)+\int_{y}^{x} C^{-}(x, s) K(s, y) d s \\
C^{-}(x, y) & =\Sigma^{+-} L(x, y)+\int_{y}^{x} C^{-}(x, s) L(s, y) d s \\
D(x) & =\Sigma^{+-} \gamma(x)+\int_{0}^{x} D(y) \gamma(y) d y \\
C_{0} & =C+Q_{0} \mathcal{K}
\end{aligned}
$$

We prove well-posedness of the kernel equations over the next two sections. First, we study a relatively general class of hyperbolic PDEs on a triangular domain.

\section{A general class of kernel equations}

\subsection{Problem setup}

We consider the following class of equations on a triangular domain

$$
\epsilon_{i}(x) \partial_{x} F_{i}(x, y)+v_{i}(y) \partial_{y} F_{i}(x, y)=\Sigma_{i}(x, y) F(x, y)
$$

where $F=\left(\begin{array}{lll}F_{1} & \cdots & F_{n}\end{array}\right)^{T}$. Each unknown $F_{i}$ satisfies boundary conditions on a subset $\Omega_{i} \subset \partial \mathcal{T}$ of the following form

$$
\forall i=1, \ldots,\left.n \quad F_{i}\right|_{\Omega_{i}}=f_{i}+\left.\sum_{j=1}^{n} \Gamma_{i j}(\cdot) F_{j}\right|_{\Omega_{i}}
$$

where $f_{i}$ and $\Gamma_{i j}$ are defined on $\Omega_{i}$. The functions $\Gamma_{i j}$, defined on the boundaries of the triangular domain $\mathcal{T}$, are boundary couplings between the different kernels $F_{i}$. The well-posedness of (44),(45) depends on the sparsity of the matrix $\Gamma=\left(\Gamma_{i j}\right)$. More precisely, consider the following definition.

Definition 3.1 Let $\mathcal{G}$ be the directed graph whose vertices are the $F_{i}$ and whose edges are defined by the matrix $\left(\left\|\Gamma_{i j}\right\|_{\infty}\right)$. In other words, there is an edge between nodes $i$ and $j$ iff $\left\|\Gamma_{i j}\right\|_{\infty} \neq 0$. Thus, a valid path of length $p$ in the graph is a $p$-uplet $a=\left(a_{1}, \ldots, a_{p}\right)$ such that

$$
\prod_{k=1}^{p-1}\left\|\Gamma_{a_{k}, a_{k+1}}\right\|_{\infty} \neq 0
$$

By convention, a path $\left(a_{1}\right)$ of length $p=1$ is the single node $F_{a_{1}}$.

The following Theorem gives a sufficient condition on the structure of $\mathcal{G}$ for the system to be well-posed.

Theorem 3.2 Consider system (44) with boundary conditions (45). Assume

(i) that the uncoupled system, obtained by taking $\Sigma(x, y) \equiv$ 0 in (44) and $\Gamma_{i j}=0, \forall i, j$ in (45), is well-posed; (ii) that there exists $\alpha>1$ such that, for all $i=1, \ldots, n$, the following inequality holds

$$
\forall(x, y) \in \mathcal{T} \quad \alpha \epsilon_{i}(x)-v_{i}(y)>\delta>0
$$

(iii) The graph $\mathcal{G}$ is acyclic, i.e. is does not contain any cycles.

Then there is a unique solution $F \in L^{\infty}(\mathcal{T})$.

Remark 1 A necessary and sufficient condition for Assumption ( $i$ ) to be satisfied is that, for every $i=1, \ldots, n$ the characteristics defined by the $\epsilon_{i}, v_{i}$ connect each point of $\mathcal{T}$ to $\Omega_{i}$.

Remark 2 Assumption (ii) is a simple geometric condition for the well-posedness of the system: the tangent vec$\operatorname{tor}\left(\epsilon_{i}(x), \mu_{i}(y)\right)$ to all the characteristics, at all points $(x, y) \in$ $\mathcal{T}$ must lie in the half-space such that the scalar product with $(\alpha,-1)^{T}$ is negative. In other words, the characteristics leaving the boundaries where (45) are defined must always "point away" from a certain line $y=\alpha x$, with $\alpha>1$. Examples of such characteristics are pictured on Figure 3.

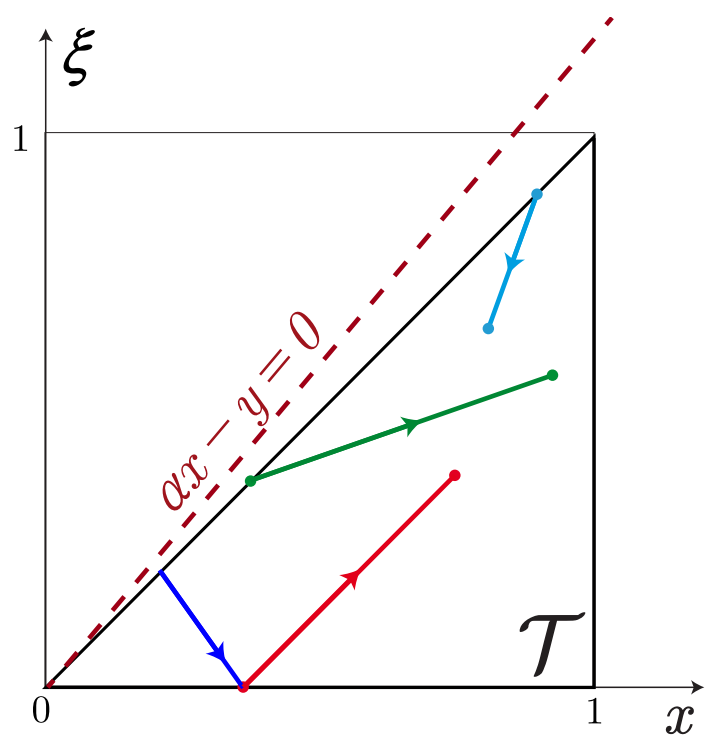

Fig. 3. Examples of characteristic lines that satisfy Assumption (ii) of Theorem 3.2.

The proof of Theorem 3.2 is quite involved and spans over the next few sections. It relies on the transformation of (44),(45) into integral equations. For this, we define in the next section the characteristic curves.

\subsection{Transformation into integral equations}

Assumption (i) of Theorem 3.2 yields the existence and uniqueness of characteristic curves, defined as follows 
Definition 3.3 For each $i=1, \ldots, n$ and any $(x, y) \in \mathcal{T}$ there exists $\left(\chi_{i}^{0}(x, y), \xi_{i}^{0}(x, y)\right) \in \Omega_{i}$ and $s_{i}^{F}(x, y) \in \mathbb{R}^{+}$such that

$$
\begin{gathered}
\left\{\begin{aligned}
\frac{d \chi_{i}(s ; x, y)}{d s} & =\epsilon_{i}\left(\chi_{i}(s ; x, y)\right) \\
\chi_{i}(0 ; x, y) & =\chi_{i}^{0}(x, y) \in \Omega_{i} \\
\chi_{i}\left(s_{i}^{F}(x, y) ; x, y\right) & =x
\end{aligned}\right. \\
\left\{\begin{aligned}
\frac{d \xi_{i}(s ; x, y)}{d s} & =v_{i}\left(\xi_{i}(s ; x, y)\right) \\
\xi_{i}(0 ; x, y) & =\xi_{i}^{0}(x, y) \in \Omega_{i} \\
\xi_{i}\left(s_{i}^{F}(x, y) ; x, y\right) & =y
\end{aligned}\right.
\end{gathered}
$$

The curves $\left(\chi_{i}(s), \xi_{i}(s)\right)$ are the characteristic curves associated with $F_{i}$. For any two points $\left(M_{1}, M_{2}\right) \in \mathcal{T}$, we denote $C_{i}\left(M_{1}, M_{2}\right)$ the characteristic curve associated with $F_{i}$ starting in $M_{1}=\left(x_{1}, y_{1}\right)$ and ending in $M_{2}=\left(x_{2}, y_{2}\right)$, if such a curve exists, i.e. if

$$
\chi_{i}\left(s^{F}\left(x_{1}, y_{1}\right) ; x_{1}, y_{1}\right)=x_{2}, \quad \xi_{i}\left(s^{F}\left(x_{1}, y_{1}\right) ; x_{1}, y_{1}\right)=y_{2}
$$

In the absence of the boundary couplings $\Gamma_{i j}$, the proof of well-posedness would consist in integrating (44) along (48),(49) and using a method of successive approximations. Here, this yields

$$
\begin{gathered}
F_{i}(x, y)=f_{i}\left(\chi_{i}^{0}(x, y), \xi_{i}^{0}(x, y)\right) \\
+\sum_{j=1}^{n} \Gamma_{i j}\left(\chi_{i}^{0}(x, y), \xi_{i}^{0}(x, y)\right) F_{j}\left(\chi_{i}^{0}(x, y), \xi_{i}^{0}(x, y)\right) \\
+\int_{0}^{s_{i}^{F}(x, y)} \Sigma_{i}\left(\chi_{i}(s ; x, y), \xi_{i}(s ; x, y)\right) F\left(\chi_{i}(s ; x, y), \xi_{i}(s ; x, y)\right) d s
\end{gathered}
$$

The second term still contains unknowns, and the method of successive approximations does not straightforwardly apply. Rather, the second term must, again, be integrated along the characteristics of the $F_{j}$ 's for which $\Gamma_{i j}$ is non-zero. This situation is depicted on Figure 3.2 for an example.

To avoid this situation repeating infinitely (infinitely many "rebounds"), we use the following basic results from Graph Theory.

\subsection{Basic results from Graph Theory}

The following Definitions and Lemmas are classical results, see e.g. [23].

Lemma 2 If $\mathcal{G}$ is acyclic, then the following holds

(a) There exist terminal nodes, i.e. there exists a set $T N \subset$ $\{1, \ldots, n\}$ such that

$$
\forall i \in T N, \forall k \in\{1, \ldots, n\} \quad \Gamma_{i k}=0
$$

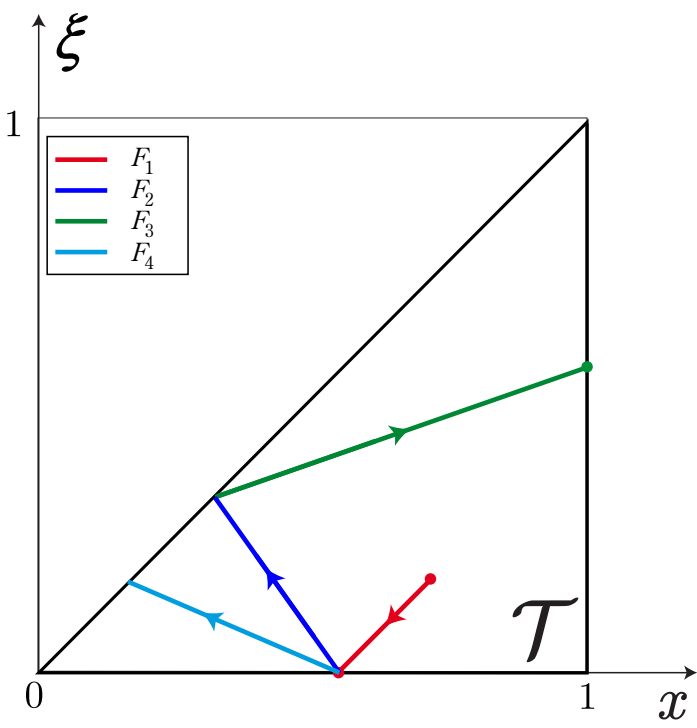

(b) All the valid paths are of finite length. In other words, any valid path can be completed with a valid path that leads to a terminal node and has a uniformely bounded length.

This allows us to add the following two definitions

Definition 3.4 For any node $F_{i}$, we define its depth $d_{i}$ as the length of the longest valid path to a terminal node. We also define $d^{\max }$ as the maximum length of any path $d^{\max }=$ $\max _{i=1, \ldots, n} d_{i}$.

Definition 3.5 Let $a=\left(a_{1}, \ldots, a_{p}\right)$ be a (not necessarily valid) path. Then, we recursively define the sequence of points $M_{k}^{a}(x, y) \in \mathcal{T}, k=0, \ldots, p$ such that

$$
\begin{aligned}
& M_{0}^{a}(x, y)=(x, y) \\
& M_{k}^{a}(x, y)=\left(\chi_{a_{k}}^{0}\left(M_{k-1}^{a}\right), \xi_{a_{k}}^{0}\left(M_{k-1}^{a}\right)\right) \in \Omega_{a_{k}}
\end{aligned}
$$

where $\chi_{a_{k}}^{0}(\cdot)$ and $\xi_{a_{k}}^{0}(\cdot)$ are defined by (48),(49). In other words, $M_{k}^{a}$ is the point on the boundary of $\mathcal{T}$ such that the characteristic curve $C_{a_{k}}\left(M_{k}^{a}, M_{k-1}^{a}\right)$ exists.

Property 3.6 For any two paths $a=\left(a_{1}, \ldots, a_{p}\right)$ and $b=$ $\left(b_{1}, \ldots, b_{q}\right)$ one has

$$
M_{p}^{a}\left(M_{q}^{b}(x, y)\right)=M_{p+q}^{(b, a)}(x, y)
$$

where $(b, a)$ denotes the concatenation of the two paths.

We are now ready to prove Theorem 3.2.

\subsection{Proof of Theorem 3.2}

Proof Classically, the proof consists in transformation the PDEs into integral equations and using a method of successive approximations. 


\subsubsection{Transformation into integral equations}

The proof relies on the following transformation of (44),(45) into integral equations. For any $i=1, \ldots, n$, any $M=(x, y) \in$ $\mathcal{T}$, one has

$$
\begin{aligned}
F_{i}(x, y) & =\sum_{p=1}^{d_{i}} \sum_{\substack{a=\left(a_{1}, \ldots a_{p}\right) \\
a_{1}=i}}\left(\prod_{k=1}^{p-1} \Gamma_{a_{k}, a_{k+1}}\left(M_{k}^{a}(x, y)\right)\right) \\
\cdot & \left(f_{a_{p}}\left(M_{p}^{a}(x, y)\right)+\int_{C_{a_{p}}\left(M_{p}^{a}(x, y), M_{p-1}^{a}(x, y)\right)} \Sigma_{a_{p}} F\right)
\end{aligned}
$$

Remark 3 The sum $\sum_{\substack{a=\left(a_{1}, \ldots a_{p}\right) \\ a_{1}=i}}$ denotes the sum over all (possibly invalid) paths of length $p$ starting from the node $F_{i}$. However, a large number of the terms of this sum is zero due to the product of $\Gamma_{a_{k}, a_{k+1}}$ inside this sum.

We now prove Equation (56) by recursion on the depth $d$.

$\mathbf{d}=1$. Consider a node $F_{i}$ such that $d_{i}=1$, i.e. $F_{i}$ is a terminal node. Assuming by convention that, the empty product is equal to 1 , i.e.

$$
\prod_{k=1}^{0} \Gamma_{a_{k}, a_{k+1}} \equiv 1
$$

Equation (56) can be rewritten as

$$
\begin{aligned}
F_{i}(x, y) & =\sum_{p=1}^{1} \sum_{\substack{a=\left(a_{1}\right) \\
a_{1}=i}} f_{a_{1}}\left(M_{1}^{a}(x, y)\right)+\int_{C_{a_{1}}\left(M_{1}^{a}(x, y), M_{0}^{a}(x, y)\right)} \Sigma_{a_{1}} F \\
& =f_{i}\left(M_{1}^{a}(x, y)\right)+\int_{C_{i}\left(M_{1}^{a}(x, y), M_{0}^{a}(x, y)\right)} \Sigma_{i} F
\end{aligned}
$$

Which exactly corresponds to integrating (44) along the characteristics associated to $F_{i}$ since for all $j=1, \ldots, n, \Gamma_{i j}$ $=0$ for a terminal node (see $(51)$ with $\left.\Gamma_{i j}=0\right)$.

$\mathbf{d} \rightarrow \mathbf{d}+\mathbf{1}$. Assume now that (56) is true for all nodes of depth less or equal to $d$, for some $d \in\left\{1, \ldots, d^{\max }\right\}$. Consider now $F_{i}$ of depth $d+1$. Integrating (44) along the characteristics and plugging in the boundary conditions (45) yields

$$
\begin{gathered}
F_{i}(x, y)=f_{i}\left(M_{1}^{(i)}(x, y)\right)+\sum_{j=1}^{n} \Gamma_{i j}\left(M_{1}^{(i)}(x, y)\right) F_{j}\left(M_{1}^{(i)}(x, y)\right) \\
+\int_{\mathcal{C}_{i}\left(M_{1}^{(i)}(x, y), M_{0}^{(i)}(x, y)\right)} \Sigma_{i} F
\end{gathered}
$$

Notice that all the $F_{j}$ for which $\Gamma_{i j} \neq 0$ are of depth $d_{j} \leq d$. Applying equation (56) to them yields

$$
\begin{gathered}
F_{i}(x, y)=f_{i}\left(M_{1}^{(i)}(x, y)\right)+\int_{C_{i}\left(M_{1}^{(i)}(x, y), M_{0}^{(i)}(x, y)\right)} \Sigma_{i} F \\
+\sum_{j=1}^{n} \sum_{p=1}^{d} \sum_{\substack{a=\left(a_{1}, \ldots a_{p}\right) \\
a_{1}=j}} \Gamma_{i j}\left(M_{1}^{(i)}(x, y)\right)\left(\prod_{k=1}^{p-1} \Gamma_{a_{k}, a_{k+1}}\left(M_{k}^{a}\left(M_{1}^{(i)}\right)\right)\right) \\
\cdot\left(f_{a_{p}}\left(M_{p}^{a}\left(M_{1}^{(i)}\right)\right)+\int_{C_{a_{p}}\left(M_{p}^{a}\left(M_{1}^{(i)}\right), M_{p-1}^{a}\left(M_{1}^{(i)}\right)\right)} \Sigma_{a_{p}} F\right)
\end{gathered}
$$

which yields

$$
\begin{gathered}
F_{i}(x, y)=f_{i}\left(M_{1}^{(i)}(x, y)\right)+\int_{C_{i}\left(M_{1}^{(i)}(x, y), M_{0}^{(i)}(x, y)\right)} \Sigma_{i} F \\
+\sum_{p=1}^{d} \sum_{j=1}^{n} \sum_{\substack{a=\left(a_{1}, \ldots a_{p}\right) \\
a_{1}=j}}\left(\Gamma_{i j}\left(M_{1}^{(i)}(x, y)\right) \prod_{k=1}^{p-1} \Gamma_{a_{k}, a_{k+1}}\left(M_{k}^{a}\left(M_{1}^{(i)}\right)\right)\right) \\
\cdot\left(f_{a_{p}}\left(M_{p}^{a}\left(M_{1}^{(i)}\right)\right)+\int_{C_{a_{p}}\left(M_{p}^{a}\left(M_{1}^{(i)}\right), M_{p-1}^{a}\left(M_{1}^{(i)}\right)\right)} \Sigma_{a_{p}} F\right)
\end{gathered}
$$

i.e.

$$
\begin{gathered}
F_{i}(x, y)=f_{i}\left(M_{1}^{(i)}(x, y)\right)+\int_{C_{i}\left(M_{1}^{(i)}(x, y), M_{0}^{(i)}(x, y)\right)} \Sigma_{i} F \\
+\sum_{p=1}^{d} \sum_{j=1}^{n} \sum_{\substack{a=\left(a_{1}, \ldots, a_{p}, a_{p+1} \\
a_{2}=j, a_{1}=i\right.}}\left(\prod_{k=1}^{p} \Gamma_{a_{k}, a_{k+1}}\left(M_{k}^{a}(x, y)\right)\right) \\
\cdot\left(f_{a_{p+1}}\left(M_{p+1}^{a}(x, y)\right)+\int_{C_{a_{p+1}}\left(M_{p+1}^{a}(x, y), M_{p}^{a}(x, y)\right)} \Sigma_{a_{p+1}} F\right)
\end{gathered}
$$

i.e.

$$
\begin{gathered}
F_{i}(x, y)=f_{i}\left(M_{1}^{(i)}(x, y)\right)+\int_{C_{i}\left(M_{1}^{(i)}(x, y), M_{0}^{(i)}(x, y)\right)} \Sigma_{i} F \\
+\sum_{p=1}^{d} \sum_{\substack{a=\left(a_{1}, \ldots, a_{p+1}\right) \\
a_{1}=i}}\left(\prod_{k=1}^{p} \Gamma_{a_{k}, a_{k+1}}\left(M_{k+1}^{a}(x, y)\right)\right) \\
\cdot\left(f_{a_{p+1}}\left(M_{p+1}^{a}(x, y)\right)+\int_{C_{a_{p+1}}\left(M_{p+1}^{a}(x, y), M_{p}^{a}(x, y)\right)} \Sigma_{a_{p+1}} F\right)
\end{gathered}
$$

i.e.

$$
\begin{aligned}
F_{i}(x, y) & =f_{i}\left(M_{1}^{(i)}(x, y)\right)+\int_{C_{i}\left(M_{1}^{(i)}(x, y), M_{0}^{(i)}(x, y)\right)} \Sigma_{i} F \\
& +\sum_{p=2}^{d+1} \sum_{\substack{a=\left(a_{1}, \ldots, a_{p}\right) \\
a_{1}=i}}\left(\prod_{k=1}^{p-1} \Gamma_{a_{k}, a_{k+1}}\left(M_{k+1}^{a}(x, y)\right)\right) \\
& \cdot\left(f_{a_{p}}\left(M_{p}^{a}(x, y)\right)+\int_{C_{a_{p}}\left(M_{p}^{a}(x, y), M_{p-1}^{a}(x, y)\right)} \Sigma_{a_{p}} F\right)
\end{aligned}
$$


i.e., using (57)

$$
\begin{aligned}
F_{i}(x, y) & =\sum_{p=1}^{d_{i}} \sum_{\substack{a=\left(a_{1}, \ldots, a_{p}\right) \\
a_{1}=i}}\left(\prod_{k=1}^{p-1} \Gamma_{a_{k}, a_{k+1}}\left(M_{k+1}^{a}(x, y)\right)\right) \\
& \left(f_{a_{p}}\left(M_{p}^{a}(x, y)\right)+\int_{C_{a_{p}}\left(M_{p}^{a}(x, y), M_{p-1}^{a}(x, y)\right)} \Sigma_{a_{p}} F\right)
\end{aligned}
$$

which concludes the proof by induction since $d_{i}=d+1$.

\subsubsection{Method of successive approximations}

The end of the proof follows the classical successive approximations method, applied to (56). More precisely, we define the following operators $\Phi=\left(\begin{array}{lll}\Phi_{1} & \cdots & \Phi_{n}\end{array}\right)^{\top}$

$$
\begin{aligned}
& \Phi_{i}[F](x, y)= \\
& \sum_{p=1}^{d_{i}} \sum_{\substack{a=\left(a_{1}, \ldots, a_{p}\right) \\
a_{1}=i}}\left(\prod_{k=1}^{p-1} \Gamma_{a_{k}, a_{k+1}}\left(M_{k}^{a}(x, y)\right)\right) \int_{\mathcal{C}_{a_{p}}\left(M_{p}^{a}(x, y), M_{p-1}^{a}(x, y)\right)} \Sigma_{a_{p}} F
\end{aligned}
$$

As well as the following vector $\phi=\left(\begin{array}{lll}\phi_{1} & \cdots & \phi_{n}\end{array}\right)^{\top}$

$$
\begin{aligned}
& \phi_{i}(x, y)= \\
& \sum_{p=1}^{d_{i}} \sum_{\substack{a=\left(a_{1}, \ldots a_{p}\right) \\
a_{1}=i}}\left(\prod_{k=1}^{p-1} \Gamma_{a_{k}, a_{k+1}}\left(M_{k}^{a}(x, y)\right)\right) f_{a_{p}}\left(M_{p}^{a}(x, y)\right)
\end{aligned}
$$

Define now the following sequence for $q \in \mathbb{N}$

$$
\begin{aligned}
\mathcal{F}^{0}(x, y)= & 0 \\
\mathcal{F}^{q+1}(x, y)= & \phi(x, y)+\Phi\left[\mathcal{F}^{q}\right](x, y) \\
= & \left(\phi_{1}(x, y) \cdots \phi_{n}(x, y)\right)^{\top}+ \\
& \left(\Phi_{1}\left[\mathcal{F}^{q}\right](x, y) \cdots \Phi_{n}\left[\mathcal{F}^{q}\right](x, y)\right)^{\top}
\end{aligned}
$$

Finally, define the following sequence for $q \geq 1$

$$
\Delta \mathcal{F}^{q}=\mathcal{F}^{q}-\mathcal{F}^{q-1}
$$

Provided the limit exists, then

$$
F=\lim _{q \mapsto+\infty} \mathcal{F}^{q}=\sum_{q=1}^{+\infty} \Delta \mathcal{F}^{q}
$$

is a solution to (56). To prove that the series is convergent, we rely on the following lemmas
Lemma 3 Assume inequality (47) holds. Then for all $i=$ $1, \ldots, n,(x, y) \in \mathcal{T}$, the following function

$$
\psi_{(x, y)}^{i}: s \in\left[0, s_{i}^{F}(x, y)\right] \mapsto \alpha \chi_{i}(s ; x, y)-\xi_{i}(s ; x, y)
$$

is strictly increasing. In particular, the following inequality holds

$$
\psi_{(x, y)}^{i}\left(s_{i}^{F}(x, y)\right)=\alpha x-y>\alpha \chi_{i}^{0}(x, y)-\xi_{i}^{0}(x, y)=\psi_{(x, y)}^{i}(0)
$$

Thus, $\psi_{(x, y)}^{i}$ defines a diffeomorphism of $\left[0, s_{i}^{F}(x, y)\right]$ onto its image $\left[\alpha \chi_{i}^{0}(x, y)-\xi_{i}^{0}(x, y), \alpha x-y\right]$.

Proof The proof is trivial since for $i=1, \ldots, n$ and $(x, y) \in \mathcal{T}$, one has

$$
\frac{d \psi_{(x, y)}^{i}}{d s}(s)=\alpha \epsilon_{i}\left(\chi_{i}(s ; x, y)\right)-v_{i}\left(\xi_{i}(s ; x, y)\right)
$$

and recalling (47).

Corollary 3.7 For any path $a=\left(a_{1}, \ldots, a_{p}\right)$ of length $p \geq 0$ and any $k=0, \ldots, p$, denote $M_{k}^{a}(x, y)=\left(x_{k}, y_{k}\right)$. Then one has

$$
\alpha x_{k}-y_{k} \leq \alpha x-y
$$

Proof We prove the result by induction. For $k=0$, given the definition of the $M_{k}^{a}$ (Equation (53)), one has

$$
\alpha x_{0}-y_{0}=\alpha x-y
$$

Assume now that (76) is satisfied for some $k=1, \ldots, p-1$, then, by definition of the $M_{k}^{a}(\cdot, \cdot)$, one has

$$
\alpha x_{k+1}-y_{k+1}=\alpha \chi_{a_{k}}^{0}\left(x_{k}, y_{k}\right)-\xi_{a_{1}}^{0}\left(x_{k}, y_{k}\right)
$$

Using Lemma 3 for $i=a_{k+1}$, this yields

$$
\alpha x_{k+1}-y_{k+1}<\alpha x_{k}-y_{k}
$$

Then, using the induction assumption, this concludes the proof.

Lemma 4 For any $i=1, \ldots, n$ and any $(x, y) \in \mathcal{T}$, one has

$$
\int_{0}^{s_{i}^{F}(x, y)}\left[\alpha \chi_{i}(s ; x, y)-\xi_{i}(s ; x, y)\right]^{q} d s \leq \frac{1}{\delta} \frac{(\alpha x-y)^{q+1}}{q+1}
$$

where $\alpha$ and $\delta$ are defined by (47).

Proof Consider the following change of variables

$$
\tau=\psi_{(x, y)}^{i}(s)
$$


where $\psi_{(x, y)}^{i}(\cdot)$ is defined by (73). It yields

$$
\begin{aligned}
& \int_{0}^{s_{i}^{F}(x, y)}\left[\alpha \chi_{i}(s ; x, y)-\xi_{i}(s ; x, y)\right]^{q} d s= \\
& \int_{\alpha \chi_{i}^{0}(x, y)-\xi_{i}^{0}(x, y)}^{\alpha x-y} \frac{\tau^{p} d \tau}{\alpha \epsilon_{i}\left(\bar{\chi}_{i}(\tau ; x, y)\right)-v_{i}\left(\bar{\xi}_{i}(\tau ; x, y)\right)}
\end{aligned}
$$

where we have abusively denoted $\bar{\chi}_{i}(\tau ; x, y)=\chi_{i}\left(\left(\psi_{(x, y)}^{i}\right)^{-1}(\tau) ; x, y\right)$ (resp. $\left.\bar{\xi}_{i}(\tau ; x, y)=\xi_{i}\left(\left(\psi_{(x, y)}^{i}\right)^{-1}(\tau) ; x, y\right)\right)$. Using (47) this yields

$$
\begin{aligned}
\int_{0}^{s_{i}^{F}(x, y)} & {\left[\alpha \chi_{i}(s ; x, y)-\xi_{i}(s ; x, y)\right]^{q} d s } \\
& <\frac{1}{\delta} \frac{[\alpha x-y]^{q+1}-\left[\alpha \chi_{i}^{0}(x, y)-\xi_{i}^{0}(x, y)\right]^{q+1}}{q+1}
\end{aligned}
$$

Since $\left(\chi_{i}^{0}(x, y), \xi_{i}^{0}(x, y)\right) \in \mathcal{T}$ and $\alpha>1$, one has $\alpha \chi_{i}^{0}(x, y)-$ $\xi_{i}^{0}(x, y)>0$ which yields the result.

Lemma 5 For any path $a=\left(a_{1}, \ldots, a_{p}\right)$ of length $p \geq 0$ and any $k=1, \ldots, p$, one has

$$
\int_{C_{a_{k}}\left(M_{k}^{a}(x, y), M_{k-1}^{a}(x, y)\right)}\left[\alpha \chi_{a_{k}}(\cdot)-\xi_{a_{k}}(\cdot)\right]^{q} \leq \frac{1}{\delta} \frac{(\alpha x-y)^{q+1}}{q+1}
$$

Proof Denoting $M_{k}^{a}(x, y)=\left(x_{k}, y_{k}\right)$, one has from Lemma 4

$$
\begin{aligned}
\int_{C_{a_{k}}\left(M_{k}^{a}(x, y), M_{k-1}^{a}(x, y)\right)}\left[\alpha \chi_{a_{k}}(\cdot)-\right. & \left.\xi_{a_{k}}(\cdot)\right]^{q} \\
& \leq \frac{1}{\delta} \frac{\left[\alpha x_{k-1}-y_{k-1}\right]^{q+1}}{q+1}
\end{aligned}
$$

Applying Corollary 3.7 yields the results.

\section{Lemma 6 Define}

$$
\begin{aligned}
\bar{\phi} & =\max _{i=1, \ldots, n}\left\|\phi_{i}(\cdot, \cdot)\right\|_{L^{\infty}(\mathcal{T})}, \quad \bar{\Gamma}=\max _{i, j \in\{1,2, \ldots, n\}}\left\|\Gamma_{i j}\right\|_{\infty} \\
\bar{\Sigma} & =\max _{(x, y) \in \mathcal{T}, i=1, \ldots, n}\left\|\Sigma_{i}(x, y)\right\| \\
M & =\frac{d^{\max }}{\delta}\left(\prod_{k=0}^{\left.d^{\max }(n-k)\right) \bar{\Gamma}^{d^{\max }-1} \bar{\Sigma}}\right.
\end{aligned}
$$

Assume that for some $q \geq 1$, one has, for all $(x, y) \in \mathcal{T}$

$$
\forall i=1, \ldots, n \quad\left|\Delta \mathcal{F}_{i}^{q}(x, y)\right| \leq \bar{\phi} \frac{M^{q}(\alpha x-y)^{q}}{q !}
$$

Then, one has

$$
\forall i=1, \ldots, n \quad\left|\Delta \mathcal{F}_{i}^{q+1}(x, y)\right| \leq \bar{\phi} \frac{M^{q+1}(\alpha x-y)^{q+1}}{(q+1) !}
$$

Assume that (89) holds for some fixed $q \geq 1$. Then, one has, for all $i=1, \ldots, n$

$$
\begin{aligned}
& \left|\Delta \mathcal{F}_{i}^{q+1}(x, y)\right| \\
& =\left|\Phi_{i}\left[\Delta \mathcal{F}^{q}\right](x, y)\right| \\
& \leq \sum_{p=1}^{d_{i}} \sum_{\substack{a=\left(a_{1}, \ldots a_{p}\right) \\
a_{1}=i}} \bar{\Gamma}^{p-1} \int_{C_{a_{p}}\left(M_{p}^{a}(x, y), M_{p-1}^{a}(x, y)\right)}\left|\Sigma_{a_{p}} \Delta \mathcal{F}^{q}\right|
\end{aligned}
$$

Using (89) yields

$$
\leq \sum_{p=1}^{d_{i}} \sum_{\substack{a=\left(a_{1}, \ldots a_{p}\right) \\ a_{1}=i}} \bar{\Gamma}^{p-1} \bar{\Sigma} \int_{C_{a_{p}}\left(M_{p}^{a}(x, y), M_{p-1}^{a}(x, y)\right)} \bar{\phi} \frac{M^{q}\left(\alpha \chi_{a_{p}}(\cdot)-\xi_{a_{p}}(\cdot)\right)^{q}}{q !}
$$

Using Lemma 5 yields

$$
\leq \sum_{p=1}^{d_{i}} \sum_{\substack{a=\left(a_{1}, \ldots a_{p}\right) \\ a_{1}=i}} \bar{\Gamma}^{p-1} \bar{\Sigma} \bar{\phi} \frac{M^{q}(\alpha x-y)^{q+1}}{(q+1) !}
$$

Noticing that there cannot be more than $\prod_{k=1}^{p}(n-k)$ paths of length $p$ from a given node $i$, this yields

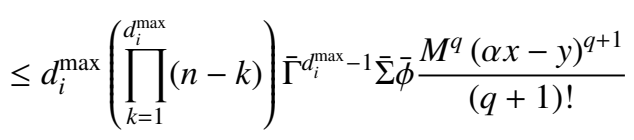

which, in turn, yields the result given the definition of $M$ (Equation (88)). Finally, Lemma 6 ensures that the series (71) is uniformly convergent, thus the kernel equations (44) with boundary conditions (45) are well-posed (see, e.g. [8] for a detailed proof). In the next section, we apply Theorem 3.2 to prove well-posedness of (31)-(38).

\section{Well-posedness of (31)-(38) and control law}

In this section, we apply the results of Section 3 to prove the well-posedness of the kernel equations. The following theorem assesses the well-posedness of the kernel equations.

Theorem 4.1 System (31)-(38) has a unique solution $K, L \in$ $L^{\infty}(\mathcal{T})$

Proof We prove the result by induction on $i=1, \ldots, n$.

$\underline{\mathbf{i}=\mathbf{1}}$. For $i=1$, the equations rewrite as follows 
for $1 \leq j \leq n$

$$
\begin{aligned}
\mu_{1} \partial_{x} K_{1 j}(x, y) & -\lambda_{j} \partial_{\xi} K_{1 j}(x, y)=-\sigma_{11}^{--} K_{1 j}(x, y) \\
& +\sum_{k=1}^{n} \sigma_{k j}^{++} K_{1 k}(x, y)+\sum_{p=1}^{m} \sigma_{p j}^{-+} L_{1 p}(x, y)
\end{aligned}
$$

for $1 \leq j \leq m$

$$
\begin{gathered}
\mu_{1} \partial_{x} L_{1 j}(x, y)+\mu_{j} \partial_{\xi} L_{1 j}(x, y)=-\sigma_{11}^{--} L_{1 j}(x, y) \\
+\sum_{p=1}^{m} \sigma_{p j}^{--} L_{1 p}(x, y)+\sum_{k=1}^{n} \sigma_{k j}^{+-} K_{1 k}(x, y) \\
\forall j, \quad K_{1 j}(x, x)=-\frac{\sigma_{1 j}^{-+}}{\mu_{1}+\lambda_{j}} \triangleq k_{1 j} \\
\forall j \neq 1, \quad L_{1 j}(x, x)=-\frac{\sigma_{1 j}^{--}}{\mu_{1}-\mu_{j}} \triangleq l_{1 j} \\
\forall j \geq 1 \quad \mu_{j} L_{1 j}(x, 0)=\sum_{k=1}^{n} \lambda_{k} K_{1 k}(x, 0) q_{k, j}+\sum_{k=1}^{p} b_{k j} \gamma_{1 k}(x) \\
\forall j, \quad \mu_{1} \gamma_{1 j}^{\prime}(x)=\sum_{k=1}^{p} a_{k j} \gamma_{1 k}(x)-\sigma_{11}^{--} \gamma_{1 j}(x) \\
\quad+\sum_{k=1}^{n} \lambda_{k} c_{k j} K^{1 k}(x, 0) \\
\forall j, \quad \gamma_{1 j}(0)=\kappa_{1 j}
\end{gathered}
$$

One can readily check that (97)-(98) are of the form (44). Besides, the ODE (103) can also be put under the form (44) by "embedding" it into $\mathcal{T}$. More precisely, denoting

$$
\mathbb{1}_{\{y=0\}}(x, y)= \begin{cases}1 & \text { if } y=0 \\ 0 & \text { otherwise }\end{cases}
$$

one can define $\tilde{\gamma}_{j}$ such that

$$
\forall(x, y) \in \mathcal{T} \quad \tilde{\gamma}_{j}(x, y)=\mathbb{1}_{\{y=0\}}(x, y) \gamma_{1 j}(x)
$$

or, equivalently, the $\tilde{\gamma}_{j}$ satisfy the following PDEs of the form (44)

$$
\begin{array}{r}
\mu_{1} \partial_{x} \tilde{\gamma}_{j}(x, y)=\mathbb{1}_{\{y=0\}}(x, y)\left[\sum_{k=1}^{n} a_{k j} \tilde{\gamma}_{k}(x, y)-\sigma_{11}^{--} \tilde{\gamma}_{j}(x, y)\right. \\
\left.+\sum_{k=1}^{n} K^{1 k}(x, y) \lambda_{k} c_{k j}\right]
\end{array}
$$

with boundary conditions

$$
\tilde{\gamma}_{j}(x, x)=\mathbb{1}_{\{y=0\}}(x, x) \kappa_{1 j}
$$

Besides, boundary conditions (99)-(101),(108) are of the form (45), with the boundary coupling coefficients $\Gamma_{i j}$ being zero for every kernel except the $L_{i j}$ on the $y=0$ boundary. Therefore, the graph defined by $\Gamma_{i j}$ is acyclic, and Theorem 3.2 applies to (97)-(104) which is well-posed, i.e. has a unique solution with $K_{1 j}, L_{1 j} \in L^{\infty}(\mathcal{T})$ and $\gamma_{1 j} \in L^{\infty}([0,1])$.

$\{\mathbf{1}, \ldots, \mathbf{i}-\mathbf{1}\} \rightarrow$ i. Let $i \in\{2, \ldots, m\}$ be fixed and assume that for $k=1, \ldots, i-1$ there exist $K_{k j}, L_{k j} \in L^{\infty}(\mathcal{T})$ and $\gamma_{k j} \in$ $L^{\infty}([0,1])$, for all $j$. Then, Equations (31)-(38) are of the form (44),(45) with coefficients in $L^{\infty}$ since they are linear in the $K_{i j}, L_{i j}$ and $\gamma_{i j}$ variables with coefficients that depend on the $K_{k j}, L_{k j}$ and $\gamma_{k j}$ for $k<j$. Thus, Theorem 3.2 applies again and the equations are well-posed.

This yields the main result of the paper, stated in the following theorem.

Theorem 4.2 Consider System (1)-(5) with the following control law

$$
\begin{aligned}
U(t)=-R_{1} u(t, 1) & +\int_{0}^{1} K(1, y) u(t, y) d y \\
& +\int_{0}^{1} L(1, y) v(t, y) d y+\gamma(1) X(t)
\end{aligned}
$$

where $K, L$ and $\gamma$ are defined by (31)-(38). Then, the zero equilibrium is exponentially stable in the $L^{2}$ sense.

Proof Theorem 4.1 ensures the existence of $K, L \in$ $L^{\infty}(\mathcal{T}), \gamma \in L^{\infty}([0,1])$ such that $(29),(30)$ holds and $(\alpha, \beta)$ satisfies (8),(12). Lemma 1 and the invertibility of the Volterra transformation yields the result.

\section{Conclusion and perspectives}

We have presented a control design for ODEs with a system of hyperbolic PDEs in the actuating path. The design results in a full-state feedback law needing measurements of the distributed actuator states along the spatial domain. This is not realistic in practice and future contributions will focus on the design of an observer solely relying on (collocated) boundary measurements.

Besides, the result opens the door to control design for other systems involving cascaded hyperbolic PDEs. In particular, networks of systems of hyperbolic balance laws are instrumental in modeling, e.g. oil production systems, networks of open channels [1] or power transmission lines [11].

\section{References}

[1] G. Bastin, J.-M. Coron, and B. d'Andréa Novel. On lyapunov stability of linearised saint-venant equations for a sloping channel. Networks and Heterogeneous Media, 4 (2):177-187, 2009.

[2] N. Bekiaris-Liberis and M. Krstic. Nonlinear Control Under Nonconstant Delays. SIAM, 2013. 
[3] N. Bekiaris-Liberis and M. Krstic. Compensation of wave actuator dynamics for nonlinear systems. Automatic Control, IEEE Transactions on, 59(6):1555-1570, June 2014.

[4] D. Bresch-Pietri. Robust control of variable time delay systems. Theoretical contributions and applications to engine control. $\mathrm{PhD}$ thesis, MINES ParisTech, 2012.

[5] D. Bresch-Pietri and M. Krstic. Output-feedback adaptive control of a wave pde with boundary anti-damping. Automatica, 50(5):14071415,2014

[6] F. Castillo Buenaventura, E. Witrant, C. Prieur, and L. Dugard. Dynamic Boundary Stabilization of Hyperbolic Systems. In Proceedings of $C D C$, page n/c, Maui, Hawaï, États-Unis, December 2012.

[7] F. Di Meglio and U. J. F. Aarsnes. A distributed parameter systems view of control problems in drilling. In to appear in 2nd IFAC Workshop on Automatic Control in Offshore Oil and Gas Production, Florianópolis, Brazil, 2015.

[8] F. Di Meglio, R. Vazquez, and M. Krstic. Stabilization of a system of $n+1$ coupled first-order hyperbolic linear pdes with a single boundary input. Automatic Control, IEEE Transactions on, 58(12):3097-3111, 2013.

[9] E. Fridman and U. Shaked. H- $\infty$ control of linear state-delay descriptor systems: an LMI approach. Linear Algebra and its Applications, 352(0):271 - 302, 2002. Fourth Special Issue on Linear Systems and Control.

[10] C. Germay, V. Denoël, and E. Detournay. Multiple mode analysis of the self-excited vibrations of rotary drilling systems. Journal of Sound and Vibration, 325(12):362 - 381, 2009.

[11] M. Günther. A pdae model for interconnected linear rlc networks. Mathematical and Computer Modelling of Dynamical Systems, 7(2):189-203, 2001.

[12] L. Hu, F. Di Meglio, R. Vazquez, and M. Krstic. Control of homodirectional and general heterodirectional linear coupled hyperbolic pdes. IEEE Transactions on Automatic Control, in review, 2015.

[13] Miroslav Krstic. Compensating actuator and sensor dynamics governed by diffusion pdes. Systems $\mathcal{E}$ Control Letters, 58(5):372377, 2009 .

[14] Miroslav Krstic and Andrey Smyshlyaev. Backstepping boundary control for first-order hyperbolic PDEs and application to systems with actuator and sensor delays. Systems Control Lett., 57(9):750758, 2008.

[15] J. Li and Y. Liu. Adaptive control of the ode systems with uncertain diffusion-dominated actuator dynamics. International Journal of Control, 85(7):868-879, 2012.

[16] W. Michiels and S. Niculescu. Stability and stabilization of timedelay systems. An eigenvalue based approach. SIAM Publications, Philadelphia, 2007, 2007.

[17] B. Ren, J.-M. Wang, and M. Krstic. Stabilization of an odeschrödinger cascade. Systems $\mathcal{E}$ Control Letters, 62(6):503 - 510, 2013.

[18] C. Sagert, F. Di Meglio, M. Krstic, and P. Rouchon. Backstepping and flatness approaches for stabilization of the stick-slip phenomenon for drilling. IFAC Sympositium on System Structure and Control, pages 779-784, 2013.

[19] M. B. Saldivar, S. Mondie, J.-J. Loiseau, and V. Rasvan. StickSlip Oscillations in Oillwell Drillstrings: Distributed Parameter and Neutral Type Retarded Model Approaches. Proceedings of the 18th IFAC World Congress, 18:284-289, 2011.

[20] O. Smith. A controller to overcome dead time. ISA Journal, 6:2833, 1959.
[21] S. Tang and C. Xie. State and output feedback boundary control for a coupled pde-ode system. Systems $\mathcal{E}$ Control Letters, 60(8):540 545,2011

[22] D. Tsubakino, T. Roux Oliveira, and M. Krstic. "predictor-feedback for multi-input lti systems with distinct delays,. In to appear in American Control Conference, 2015.

[23] Douglas Brent West et al. Introduction to graph theory, volume 2. Prentice hall Upper Saddle River, 2001.

[24] Huai-Ning Wu and Jun-Wei Wang. Observer design and output feedback stabilization for nonlinear multivariable systems with diffusion pde-governed sensor dynamics. Nonlinear Dynamics, 72(3):615-628, 2013.

[25] D. Yue and Q.-L. Han. Delayed feedback control of uncertain systems with time-varying input delay. Automatica, 41:233-240, 2005. 